\title{
De novo CD5-positive Diffuse Large B-cell Lymphoma Primarily Presenting as Spinal Cord Compression: A Case Report

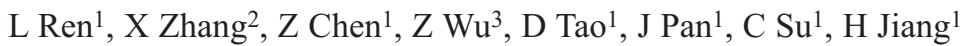

\begin{abstract}
De novo CD5-positive (CD5 $)$ diffuse large B-cell lymphoma (DLBCL) is a highly aggressive subtype of DLBCL with a high incidence of misdiagnosis when spinal cord compression is the initial symptom. We report a case of a 45-year old man with de novo $C D 5^{+} D L B C L$ primarily presenting as spinal cord compression. Unfortunately, this patient was resistant to rituximab-based therapy, which indicated a poor prognosis.
\end{abstract}

Keywords: CD5-positive diffuse large B-cell, lymphoma, spinal cord compression

WIMJ Open 2015; 2 (2): 106

\section{INTRODUCTION}

De novo CD5-positive $\left(\mathrm{CD}^{+}\right)$diffuse large $\mathrm{B}$-cell lymphoma (DLBCL) is a distinct subgroup of DLBCL associated with a high frequency of extranodal involvement and poor prognosis, accounting for approximately $10 \%$ of all DLBCLs (1$3)$. Spinal cord compression is relatively rare as the initial symptom of lymphomas (4), and can cause lymphomas to be easily misdiagnosed as bone metastatic tumours before aspiration biopsy. Here, we report the case of a patient with de novo $\mathrm{CD}^{+}$DLBCL involving the $11^{\text {th }}$ thoracic spine (T11) who primarily presented with paraplegia.

\section{CASE REPORT}

A 45-year old man experienced pain in the hips without relief after rest on August 1, 2012, with subsequent development of motor and sensory dysfunction leading to abnormalities in posture and gait that were noticed on September 22, 2012. Magnetic resonance imaging (MRI) of the thoracic spine, performed immediately, revealed an abnormal signal in the canalis vertebralis of the T11 vertebral body (Fig. 1A-B).

At the same time, 18F-fluorodeoxyglucose positron emission tomography/computed tomography ( ${ }^{18} \mathrm{FDG}-$ $\mathrm{PET} / \mathrm{CT}$ ) was also performed; increased uptake was observed at numerous foci with bone destruction in various locations throughout the body (Fig. 1C-D). For diagnosis and treat-

From: ${ }^{1}$ Department of Hematology, Tongde Hospital of Zhejiang Province, Hangzhou 310012, China, ${ }^{2}$ Shanghai Jiao Tong University School of Medicine, Shanghai 200025, China and ${ }^{3}$ Zhejiang Chinese Medical University, Hangzhou 310053, China.

Correspondence: Dr H Jiang, Department of Hematology, Tongde Hospital of Zhejiang Province, Hangzhou 310012, China. Fax: +86-0571-87236702, e-mail: jianghuifang501@163.com
A

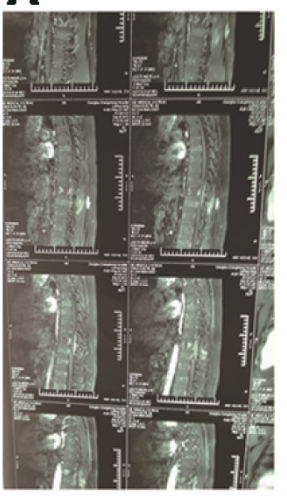

C

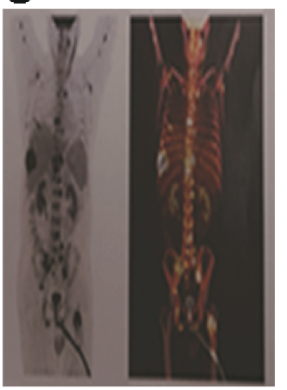

D

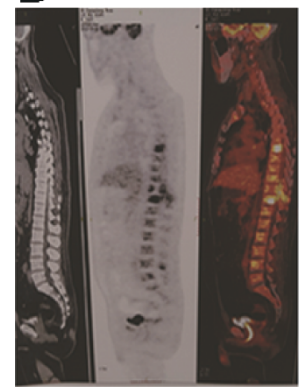

Fig. 1: A-B): Magnetic resonance imaging of the thoracic spine shows an abnormal signal in the canalis vertebralis of the $11^{\text {th }}$ thoracic vertebral body (T11). C-D): Positron emission tomography/ computed tomography shows increased uptake at numerous foci with bone destruction at various locations of the entire body including the T11 vertebral body.

ment, a mass excision surgery and vertebral reconstruction combined with internal fixation were performed on September 27, 2012; however, the spinal cord compression symptoms were not alleviated. Unfortunately, postoperative 
pathological examination of the T11 vertebral body and its accessories revealed a DLBCL, and immunohistochemical tests showed a $\mathrm{CD}^{+} \mathrm{CD} 19^{+} \mathrm{CD} 20^{++} \mathrm{CD} 79 \mathrm{a}^{++} \mathrm{CD} 23^{-}$ phenotype with $\mathrm{BCL}^{+} 2^{+}, \mathrm{C}-\mathrm{MYC}^{-}$and a $\mathrm{Ki}-67$ index of $60 \%-80 \%$. Therefore, this patient was referred to the Department of Hematology, Tongde Hospital of Zhejiang Province, for further management on October 20, 2012.

Apart from paraplegia, the patient also had a fever with the highest temperature recorded at approximately $39{ }^{\circ} \mathrm{C}$ daily, night sweats, and weight loss for nearly one month. On physical examination, two tender, firm and fixed masses of 2 $\times 3 \mathrm{~cm}$ and $5 \times 4 \mathrm{~cm}$ were palpated on the left and right arcus costarum, respectively, but no enlarged lymph nodes were palpable. A neurological examination indicated that the strength of the lower extremities was $0 / 5$ on both sides, with weakened muscle tone and absence of superficial and deep sensations.

On laboratory examination, moderate anaemia with a haemoglobin level of $8.9 \mathrm{~g} / \mathrm{L}$ and slight leucopenia $(3.5 \times$ $\left.10^{9} / \mathrm{L}\right)$ were detected. Additionally, the lactate dehydrogenase (LDH) level was within the normal range, but the alkaline phosphatase level was elevated at 299 U/L (reference range, 40-110 U/L). Bone marrow (BM) aspiration and biopsy was performed on the posterior superior iliac spine, and $\mathrm{BM}$ involvement by B-cell lymphoma was confirmed (Fig. 2A).

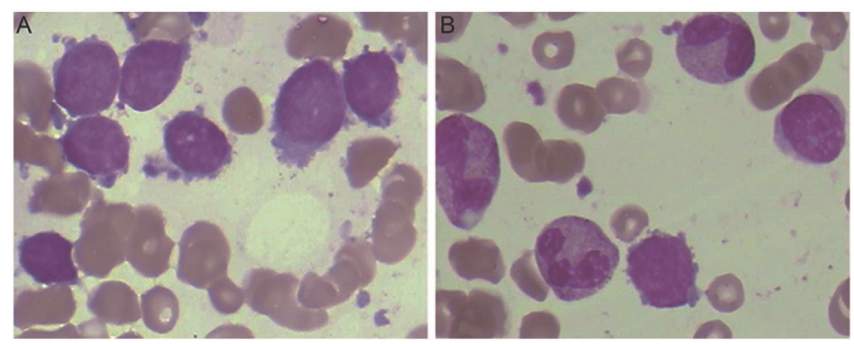

Fig. 2: A): Diffuse large B-cell lymphoma with bone marrow (BM) involvement was confirmed by BM aspiration. B): After three cycles of chemotherapy, the number of lymphoma cells in BM decreased (oil immersion, 100× magnification).

Flow cytometry analysis showed that the lymphoma cells mainly had a $\mathrm{CD}^{+} \mathrm{CD} 19^{+} \mathrm{CD} 20^{+} \mathrm{CD} 22^{+} \mathrm{CD} 38^{+} \mathrm{HLA}-$ $\mathrm{DR}^{+}$phenotype, in accordance with the phenotype expressed by lymphoma cells in the tumour mass. Therefore, de novo $\mathrm{CD}^{+}$DLBCL (stage IVB, International Prognostic Index $=3$ ) was diagnosed, with concurrent involvement of $\mathrm{BM}$ and bones.

A 21-day rituximab-cyclophosphamide doxorubicinvincristine-prednisone regimen (R-CHOP21), rituximabmethotrexate-vindesine-etoposide-dexamethasone $(\mathrm{R}+\mathrm{MTX}+\mathrm{VPE})$, and two cycles of rituximab-methotrexatecytarabine (R-HyperCVAD-B) were initiated in a cyclical schedule. However, complete remission was not achieved (Fig. 2B) in the BM, and subsequently, the patient's disease progressed and he died at the end of four cycles of chemotherapy.

\section{DISCUSSION}

De novo $\mathrm{CD}^{+}$DLBCL is associated with older age and is predominantly seen in women. An advanced clinical stage and a high frequency of BM and central nervous system involvement are also associated with this condition, representing disease aggressiveness (1). Primary CD5 ${ }^{+}$ DLBCL can involve several organs including the heart (5), temporal bone (6), adrenal glands (7), liver and spleen (2), but spinal cord compression as the initial presentation is rarely associated with this subtype. Solid tumours such as lung cancer, ovarian cancer and prostatic cancer are associated with spinal involvement more frequently than lymphomas, and therefore, there is a high possibility of misdiagnosis. Symptoms associated with the primary focus and an elevated level of tumour markers is readily observed when solid tumours result in spinal cord compression. However, lymphoma is usually characterized by multifocal lymphadenectasis; systemic B symptoms including persistent fever, night sweats and weight loss; a high LDH level and rapid involvement of other non-lymphoid organs and tissues, differentiating it from spinal metastasis from solid tumours. Bone involvement in cases of lymphoma is confirmed by histological examination and immunophenotyping, while MRI and PET/CT can contribute to early detection of foci and evaluation of the entire body, including nodal and extranodal sites, respectively. In our patient, spinal cord compression with progressive dyskinesia of the lower extremities without palpable superficial lymph nodes was the initial presentation, but systemic B symptoms rapidly developed and involvement of multiple bone sites was observed on PET/CT; because of this, we considered the possibility of a lymphoma.

Generally, apart from the initial biopsy to establish a diagnosis or the treatment strategy for pathological fractures, surgery is avoided in the management of bone involvement (8), but this guideline is challenged in cases of DLBCLassociated spinal cord compression, where surgical decompression could improve neurological deficit and provide a survival benefit (9). This patient underwent mass excision surgery to acquire tissue specimens for diagnosis and vertebral reconstruction combined with internal fixation for the treatment of spinal cord compression. A recent study showed that overall survival and progression-free survival do not differ in patients of stage IV DLBCL with or without bone involvement $(8,10)$. Treatment with rituximab improves the five-year overall survival (59\%) and progression-free survival (54\%) rates of patients with DLBCL with bone involvement, and its therapeutic effect is almost equal to that in DLBCL without bone involvement $(8,10)$. However, these rates are quite different for cases of CD5+ DLBCL, with the two-year overall survival rate ranging from $22.1 \%$ to $59.0 \%$, regardless of bone involvement $(1,3)$. CD5positive DLBCL is resistant to conventional chemotherapy and rituximab-based therapy (3), and although R-CHOP21, $\mathrm{R}+\mathrm{MTX}+\mathrm{VPE}$ and R-Hyper-CVAD regimens were initiated 
in sequence, complete remission was not achieved in BM, indicating a poor prognosis. Enrolment in clinical trials should be considered when standard regimens are ineffective.

Taken together, our results suggest that lymphoma should be considered in cases of spinal cord involvement by tumours, especially when systemic symptoms and enlarged lymph nodes are observed. Nevertheless, a conclusive diagnosis is based on histopathological examination. CD5positive DLBCL is an aggressive subtype of DLBCL, associated with a poor prognosis, and enrolment in clinical trials should be considered when rituximab-based therapy is not applicable.

\section{ACKNOWLEDGEMENTS}

The authors thank the Tongde Hospital of Zhejiang Province for their kind assistance. The authors declare no conflict of interest.

\section{REFERENCES}

1. Zhang Q, Zhang HY, Zhong FL, Zhang WL, Xu L, Feng J et al. Clinical analysis of 10 patients with de novo CD5 positive diffuse large B cell lymphoma. J Exp Hematol 2013; 21: 399-402.

2. Zhang X, Sun M, Zhang L, Shao H. Primary hepatosplenic CD5positive diffuse large B-cell lymphoma: a case report with literature review. Int J Clin Exp Pathol 2013; 6: 985-9.

3. Hyo R, Tomita N, Takeuchi K, Aoshima T, Fujita A, Kuwabara H et al. The therapeutic effect of rituximab on CD5-positive and CD5-negative diffuse large B-cell lymphoma. Hematol Oncol 2010; 28: 27-32.

4. Chahal S, Lagera JE, Ryder J, Kleinschmidt-DeMasters BK Hematological neoplasms with first presentation as spinal cord compression syndromes: a 10-year retrospective series and review of the literature. Clin Neuropathol 2003; 22: 282-90.
5. Cioc AM, Jessurun J, Vercellotti GM, Pambuccian SE. De novo CD5positive primary cardiac diffuse large B-cell lymphoma diagnosed by pleural fluid cytology. Diagn Cytopathol 2014; 42: 259-67. doi: 10.1002/dc.22918. Epub 2012 Sep 25.

6. Ogawa S, Tawara I, Ueno S, Kimura M, Miyazaki K, Nishikawa $\mathrm{H}$ et al. De novo CD5-positive diffuse large B-cell lymphoma of the temporal bone presenting with an external auditory canal tumor. Intern Med 2006; 45: 733-7.

7. Rashidi A, Bergeron CW, Fisher SI, Chen IA. Primary adrenal de novo CD5-positive diffuse large B cell lymphoma. Ann Hematol 2013; 92: $1281-2$.

8. Lee HY, Kim SJ, Kim K, Ko YH, Kim WS. Bone involvement in patients with stage IV diffuse large B-cell lymphoma: does it have a prognostic value? Leuk Lymphoma 2012; 53: 173-5.

9. Chang CM, Chen HC, Yang Y, Wang RC, Hwang WL, Teng CL. Surgical decompression improves recovery from neurological deficit and may provide a survival benefit in patients with diffuse large B-cell lymphoma-associated spinal cord compression: a case-series study. World J Surg Oncol 2013; 11: 90.

10. Tang Y, Yang X, Xiao J, Liu K, Yan W, Song D et al. Clinical outcomes of treatment for spinal cord compression due to primary non-Hodgkin lymphoma. Spine J 2013; 13: 641-50.

Received 21 Jan 2015

Accepted 05 Feb 2015

Published 29 May 2015

Online: http://www.mona.uwi.edu/wimjopen/article/1637

(C) Ren et al 2015

This is an open access article made freely available under Creative Commons Attribution 4.0 International (CC BY 4.0). Users are free to share, copy and adapt this work as long as the copyright holder (author) is appropriately and correctly credited. See http://creativecommons.org/ licences/by/4.0/deed.en_us for more information. 